\title{
ASYMPTOTIC BEHAVIOUR FOR A PARTIALLY DIFFUSIVE RELAXATION SYSTEM
}

\author{
BY \\ MIGUEL ESCOBEDO (Departamento de Matemáticas, Universidad del País Vasco, Apartado 644, \\ E-48080 Bilbao, Spain) \\ AND
}

PHILIPPE LAURENÇOT (Mathématiques pour l'Industrie et la Physique, CNRS UMR 5640, Université Paul Sabatier - Toulouse 3, 118 route de Narbonne, F-31062 Toulouse cedex 4, France)

\begin{abstract}
The time asymptotics of nonnegative and integrable solutions to a partially diffusive relaxation system is investigated. Under suitable assumptions on the relaxation term, the convergence to a self-similar source type solution, either of the heat equation or of the viscous Burgers equation, is proved. The proof relies on optimal decay rates and classical scaling arguments.
\end{abstract}

1. Introduction. We study the large time behaviour of nonnegative and integrable solutions to the system

$$
\begin{aligned}
& u_{t}+u_{x}-u_{x x}=k\left(v-u^{q}\right) \text { in }(0,+\infty) \times \mathbb{R}, \\
& v_{t}=k\left(u^{q}-v\right) \text { in }(0,+\infty) \times \mathbb{R},
\end{aligned}
$$

with initial data

$$
(u, v)(0)=\left(u_{0}, v_{0}\right) \text { in } \mathbb{R},
$$

where $k$ and $q$ are positive real numbers and $u_{0}, v_{0}$ are nonnegative, bounded and integrable functions on $\mathbb{R}$. A first step towards the understanding of the large time behaviour of integrable solutions to (1)-(2) is performed in [GvDD], where a generalized version of (1)-(2) is considered as a model for the one-dimensional transport of a one-species contaminant through a porous medium accounting for adsorption reactions. Assuming the reaction to be fast, that is, $k \rightarrow+\infty$, the system (1)-(2) formally reduces to

$$
\left(u+u^{q}\right)_{t}+u_{x}-u_{x x}=0 \text { in }(0,+\infty) \times \mathbb{R} .
$$

Let us also mention that the fast reaction limit $k \rightarrow \infty$ for the system without the diffusive term $u_{x x}$ has been considered in $[\mathrm{KT}]$ and [TW] for one space dimension, and in $[\mathrm{KaTz}]$ for general space dimension.

Received August 27, 2001.

2000 Mathematics Subject Classification. Primary 35L45, 35B40, 35K55.

(C)2003 Brown University 
Asymptotic expansions of solutions to (3) as $t \rightarrow \infty$ are constructed in [GvDD] according to the different values of $q$, while analytic results are provided in [EZ, EVZ, LS, Re]. Summarizing the results obtained in the above mentioned papers, five cases are to be distinguished: if $q \in(0,1), u^{q}(t)$ converges in $L^{1}(\mathbb{R})$ to the self-similar source-type solution to the scalar conservation law $z_{t}-\left(z^{q}\right)_{x}=0$ with total mass $M:=\left|u_{0}+u_{0}^{q}\right|_{L^{1}}$, while (3) is a linear equation for $q=1$ whose large time behaviour follows at once from that of the linear heat equation. Introducing $U(t, x)=u(t, x+t), U(t)$ converges to $L^{1}(\mathbb{R})$ to the self-similar source-type solution to $z_{t}-\left(z^{q}\right)_{x}=0, z_{t}-\left(z^{2}\right)_{x}-z_{x x}=0$ and $z_{t}-z_{x x}=0$ with total mass $M$ when $q \in(1,2), q=2$, and $q>2$, respectively. We refer to the above mentioned papers for a more precise description of the large time behaviour of solutions to $(3)$.

A possible expectation is that the study of the large time behaviour of solutions to (3) gives some clues towards the description of the large time behaviour of solutions to (1)-(2) for finite values of $k$, at least for some values of the parameter $q$. This is actually what we aim to prove in this paper when $q$ ranges in $[2,+\infty)$. In view of the results already known for (3) when $q \in[2,+\infty)$, the large time behaviour of the first component $u$ of the solutions to (1)-(2) is expected to be ruled by the diffusion term if $q>2$, while it is given by a balance between diffusion and convection for $q=2$. More precisely, our result reads as follows:

TheOREm 1. Assume that $q \in[2,+\infty)$ and consider two measurable functions $u_{0}$ and $v_{0}$ satisfying

$$
\left(u_{0}, v_{0}\right) \in L^{1}\left(\mathbb{R} ; \mathbb{R}^{2}\right) \cap L^{\infty}\left(\mathbb{R} ; \mathbb{R}^{2}\right), u_{0}, v_{0} \geq 0 \text { a.e. in } \mathbb{R} .
$$

Let $(u, v)$ be the solution to (1)-(2) with initial data $\left(u_{0}, v_{0}\right)$. Introducing $U(t, x)=$ $u(t, x+t)$ for $(t, x) \in(0,+\infty) \times \mathbb{R}$ and $M=\left|u_{0}+v_{0}\right|_{L^{1}}$, there holds

$$
\lim _{t \rightarrow+\infty} t^{(1-1 / p) / 2}\left|U(t)-S_{M}(t)\right|_{L^{p}}=0
$$

for $p \in[1, \infty)$, where

a: $S_{M}$ is the unique nonnegative solution to

$$
\begin{aligned}
S_{M, t}-S_{M, x x} & =0 \text { in }(0,+\infty) \times \mathbb{R}, \\
S_{M}(0) & =M \delta
\end{aligned}
$$

if $q>2$, and

b: $S_{M}$ is the unique nonnegative solution to

$$
\begin{aligned}
S_{M, t}-\left(S_{M}^{2}\right)_{x}-S_{M, x x} & =0 \text { in }(0,+\infty) \times \mathbb{R}, \\
S_{M}(0) & =M \delta
\end{aligned}
$$

if $q=2$.

Here $\delta$ denotes the Dirac mass centered at $x=0$.

The description of the long time behaviour of the solutions to (1)-(2) is an open problem when $q<2$. According to the formal analysis in [GvDD], the diffusive effects are expected to vanish as $t \rightarrow \infty$ and our approach does not allow to handle this case. 
REMARK 1. For the sake of completeness, we briefly recall that for every fixed $M>0$, the functions $S_{M}$ defined by (6) and (7) are given by

$$
S_{M}(t, x)=t^{-1 / 2} f_{M}\left(x t^{-1 / 2}\right), \quad \int_{-\infty}^{+\infty} f_{M}(z) d z=M
$$

with

$$
f_{M}(z)=C_{M} e^{-|z|^{2} / 4} \text { for }(6)
$$

and

$$
f_{M}(z)=e^{-|z|^{2} / 4}\left\{C_{M}+\int_{-\infty}^{z} e^{-|s|^{2} / 4} d s\right\}^{-1} \text { for (7), }
$$

where $C_{M}$ is a positive constant such that the total mass of $f_{M}$ is $M$.

Before proceeding with the proof of Theorem 1, let us briefly describe the main steps. First we may take $k=1$ without loss of generality. Following the analysis of [GvDD] we first perform a temporal shift on the space variable by putting $(U, V)(t, x)=(u, v)(t, x+t)$ for $(t, x) \in(0,+\infty) \times \mathbb{R}$. Then $(U, V)$ solves

$$
U_{t}-U_{x x}=V-U^{q}, \quad V_{t}-V_{x}=U^{q}-V .
$$

As $U$ is expected to become invariant with respect to a suitable scaling of the time and space variables for large times, we shall employ a scaling method to prove Theorem 1. Scaling arguments have been widely used in the study of the large time behaviour of parabolic and hyperbolic equations (see, e.g., [Va, LP] and the references therein). More precisely, in view of the expected result, we introduce

$$
U_{\lambda}(t, x)=\lambda U\left(\lambda^{2} t, \lambda x\right) \text { and } V_{\lambda}(t, x)=\lambda^{q} V\left(\lambda^{2} t, \lambda x\right)
$$

for $(t, x) \in(0,+\infty) \times \mathbb{R}$ and $\lambda \geq 1$. Notice that the functions $S_{M}$ defined above are invariant under the scaling performed on $U$. The pair $\left(U_{\lambda}, V_{\lambda}\right)$ then satisfies the following system:

$$
\begin{aligned}
V_{\lambda}-U_{\lambda}^{q} & =\lambda^{-1} V_{\lambda, x}-\lambda^{-2} V_{\lambda, t}, \\
U_{\lambda, t}-U_{\lambda, x x} & =\lambda^{2-q} V_{\lambda, x}-\lambda^{1-q} V_{\lambda, t} .
\end{aligned}
$$

We now formally pass to the limit as $\lambda \rightarrow+\infty$ in the previous system and conclude that a possible limit $\left(U_{\infty}, V_{\infty}\right)$ of $\left(U_{\lambda}, V_{\lambda}\right)$ should satisfy $V_{\infty}=U_{\infty}^{q}$, the function $U_{\infty}$ being a solution to (6) if $q>2$ or to (7) if $q=2$. In addition, the choice of the scaling performed on $(U, V)$ warrants that $U_{\infty}(0)=\left(\left|u_{0}+v_{0}\right|_{L^{1}}\right) \delta$, which allows to uniquely determine $U_{\infty}$. A rigorous justification of this formal limiting procedure is the purpose of this paper and requires the boundedness of $\left(U_{\lambda}, V_{\lambda}\right)$ in suitable norms, or equivalently, optimal temporal decay rates for various norms of $(U, V)$. After collecting some preliminary results in Sec. 2 , Sec. 3 is devoted to the proof of temporal decay estimates for $(U, V)$. The proof of Theorem 1 is performed in the last section.

REMARK 2. System (1)-(2) without the diffusive term, i.e.,

$$
\begin{aligned}
u_{t}+u_{x} & =k\left(v-u^{q}\right) \text { in }(0,+\infty) \times \mathbb{R}, \\
v_{t} & =k\left(u^{q}-v\right) \text { in }(0,+\infty) \times \mathbb{R},
\end{aligned}
$$

is a particular case of hyperbolic systems with relaxation considered by T.-P. Liu [L] (see $[\mathrm{Na}]$ for a recent review on the subject). As this author pointed out, under the strict 
subcharacteristic condition, the large time behaviour of these systems is described by a nonlinear diffusion equation. This has been proved subsequently for some particular cases (cf. $[\mathrm{C}, \mathrm{LN}]$ and the references therein).

Let us notice here that the strict subcharacteristic condition is not fulfilled by (9)-(10). Actually, the same formal computation as in [L, Sec. 2] indicates that $W=(u+v)(t, x+t)$, behaves, as $t \rightarrow \infty$, like the solutions to

$$
w_{t}-\left(w^{q}\right)_{x}-\left(w^{q}\right)_{x x}=0,
$$

and therefore, by the results proved in [LS], is asymptotic to integrable self-similar solutions to the hyperbolic equation

$$
w_{t}-\left(w^{q}\right)_{x}=0 .
$$

This also indicates that the large time behaviour of the system (1)-(2) and its inviscid counterpart (9)-(10) are of a different nature.

2. Well-posedness and basic properties. In this section we assume that $q>1$ and denote by $\widetilde{G}(t)$ the linear semigroup in $L^{1}(\mathbb{R})$ generated by the operator $\left(\partial_{x x}-\partial_{x}\right)$. We first recall the well-posedness of (1)-(2) for initial data satisfying (4).

Proposition 2. Let $\left(u_{0}, v_{0}\right)$ be two measurable functions satisfying (4). There is a unique couple of nonnegative functions

$$
(u, v) \in \mathcal{C}\left([0,+\infty) ; L^{1}\left(\mathbb{R} ; \mathbb{R}^{2}\right)\right) \cap L^{\infty}\left((0,+\infty) \times \mathbb{R} ; \mathbb{R}^{2}\right)
$$

such that $(u, v)$ satisfies

$$
\begin{aligned}
& u(t)=\widetilde{G}(t) u_{0}+\int_{0}^{t} \widetilde{G}(t-s)\left(v-u^{q}\right)(s) d s, \\
& v(t)=e^{-t} v_{0}+\int_{0}^{t} e^{s-t} u^{q}(s) d s
\end{aligned}
$$

for $t \in[0,+\infty)$. In addition,

$$
\int(u(t, x)+v(t, x)) d x=\int\left(u_{0}(x)+v_{0}(x)\right) d x, t \in[0,+\infty) .
$$

For further use we introduce the notation $(u(t), v(t))=S_{t}\left(u_{0}, v_{0}\right)$ for $t \geq 0$.

REMARK 3. It actually follows from (11) and (14) that the right-hand side of (1) belongs to $L^{\infty}\left(0,+\infty ; L^{p}(\mathbb{R})\right)$ for every $p \in(1, \infty)$. Classical parabolic regularity results [LSU] then entail that $u \in W_{p}^{1,2}((0, T) \times \mathbb{R})$ for each $p \in(1, \infty)$ and $T \in(0,+\infty)$. Consequently, $u$ is a strong solution to (1).

We next notice that the right-hand side of (1)-(2) is quasi-monotone which ensures that the mapping $S_{t}$ is an order-preserving contraction in $L^{1}\left(\mathbb{R} ; \mathbb{R}^{2}\right)$ (see, e.g., [Na, Sec. $5])$. That is, if $\left(u_{0}, v_{0}\right)$ and $\left(\hat{u}_{0}, \hat{v}_{0}\right)$ are two pairs of functions satisfying (4), there holds

$$
\left|(u-\hat{u})_{+}(t)\right|_{L^{1}}+\left|(v-\hat{v})_{+}(t)\right|_{L^{1}} \leq\left|\left(u_{0}-\hat{u}_{0}\right)_{+}\right|_{L^{1}}+\left|\left(v_{0}-\hat{v}_{0}\right)_{+}\right|_{L^{1}}
$$

for $t \in[0,+\infty)$, with the obvious notation $(u, v)(t)=S_{t}\left(u_{0}, v_{0}\right),(\hat{u}, \hat{v})(t)=S_{t}\left(\hat{u}_{0}, \hat{v}_{0}\right)$, and $r_{+}=\max \{r, 0\}$. As a consequence of (14)--(15) and [CT], we have

$$
|(u-\hat{u})(t)|_{L^{1}}+|(v-\hat{v})(t)|_{L^{1}} \leq\left|\left(u_{0}-\hat{u}_{0}\right)\right|_{L^{1}}+\left|\left(v_{0}-\hat{v}_{0}\right)\right|_{L^{1}}
$$


for every $t \in[0,+\infty)$ and pairs $\left(u_{0}, v_{0}\right),\left(\hat{u}_{0}, \hat{v}_{0}\right)$ satisfying (4). Observe finally that, thanks to $(16)$, we may extend $S_{t}$ by density to the whole positive cone of $L^{1}\left(\mathbb{R} ; \mathbb{R}^{2}\right)$.

We now fix a couple $\left(u_{0}, v_{0}\right)$ of functions satisfying (4). For $t \in[0,+\infty)$ we put $(u(t), v(t))=S_{t}\left(u_{0}, v_{0}\right)$ and

$$
M:=\int\left(u_{0}+v_{0}\right)(x) d x .
$$

We first recall that (14) and the nonnegativity of $u$ and $v$ entail

$$
|u(t)|_{L^{1}}+|v(t)|_{L^{1}} \leq M, \quad t \in[0,+\infty) .
$$

The remainder of this section is devoted to the analysis of some properties enjoyed by $(u, v)$.

LEmma 3. There is a constant $C_{1}$ depending only on $q$ and $M$ such that, for each $p \in[0,+\infty), t_{1} \in(0,+\infty)$, and $t_{2} \in\left(t_{1},+\infty\right)$, there holds

$$
\begin{aligned}
& \left|u\left(t_{2}\right)\right|_{L^{1+p q}}^{1+p q}+\frac{1+p q}{1+p}\left|v\left(t_{2}\right)\right|_{L^{1+p}}^{1+p}+\frac{1}{C_{1}} \frac{p q}{1+p q} \int_{t_{1}}^{t_{2}}|u(s)|_{L^{\infty}}^{2+p q} d s \\
& \leq\left|u\left(t_{1}\right)\right|_{L^{1+p q}}^{1+p q}+\frac{1+p q}{1+p}\left|v\left(t_{1}\right)\right|_{L^{1+p}}^{1+p} .
\end{aligned}
$$

Proof. Let $p \in[0,+\infty)$. We multiply (1) by $u^{p q},(2)$ by $v^{p}$, add the resulting identities and integrate over $\left(t_{1}, t_{2}\right) \times \mathbb{R}$; we obtain

$$
\begin{aligned}
& \left|u\left(t_{2}\right)\right|_{L^{1+p q}}^{1+p q}+\frac{1+p q}{1+p}\left|v\left(t_{2}\right)\right|_{L^{1+p}}^{1+p} \\
& +\int_{t_{1}}^{t_{2}} \int\left(v-u^{q}\right)\left(v^{p}-u^{p q}\right) d x d s+\frac{4 p q}{1+p q} \int_{t_{1}}^{t_{2}}\left|\left(u^{(1+p q) / 2}\right)_{x}\right|^{2} d x d s \\
& \leq\left|u\left(t_{1}\right)\right|_{L^{1+p q}}^{1+p q}+\frac{1+p q}{1+p}\left|v\left(t_{1}\right)\right|_{L^{1+p}}^{1+p} .
\end{aligned}
$$

By the Gagliardo-Nirenberg inequality and (18), we have

$$
\begin{aligned}
|u|_{L^{\infty}}^{(1+p q) / 2} & \leq C_{1}\left|\left(u^{(1+p q) / 2}\right)_{x}\right|_{L^{2}}^{1 / 2}\left|\left(u^{(1+p q) / 2}\right)\right|_{L^{2}}^{1 / 2} \\
& \leq C_{1}|u|_{L^{1}}^{1 / 4}|u|_{L^{\infty}}^{p q / 4}\left|\left(u^{(1+p q) / 2}\right)_{x}\right|_{L^{2}}^{1 / 2} \\
|u|_{L^{\infty}}^{2+p q} & \leq C_{1}\left|\left(u^{(1+p q) / 2}\right)_{x}\right|_{L^{2}}^{2} .
\end{aligned}
$$

The inequality (19) then follows at once from (20) and (21).

We next exploit further (19) to obtain a differential inequality involving various $L^{p_{-}}$ norms of $u$ and $v$ which will lead to the expected temporal decay estimates (see Lemma 6 and Lemma 8 below).

LEMmA 4. There are positive constants $C_{2}$ and $C_{3}$ depending only on $q$ and $M$ such that, if $p \geq(q-3)_{+} / q$, the mapping

$$
t \mapsto E_{p}(t):=|u(t)|_{L^{1+p q}}^{1+p q}+\frac{1+p q}{1+p}|v(t)|_{L^{1+p}}^{1+p}+\frac{C_{2} p q}{1+p q}|v(t)|_{L^{p+3 / q}}^{p+3 / q}
$$


is nonincreasing and satisfies

$$
\frac{p q}{1+p q} \int_{t_{1}}^{t_{2}} D_{p}(s) d s \leq C_{3} E_{p}\left(t_{1}\right), \quad 0 \leq t_{1} \leq t_{2}
$$

with

$$
D_{p}(s)=|u(s)|_{L^{\infty}}^{2+p q}+|v(s)|_{L^{p+3 / q}}^{p+3 / q} .
$$

Proof. We multiply (2) by $v^{p-1+3 / q}$ and integrate over $\left(t_{1}, t_{2}\right) \times \mathbb{R}$; using the Young inequality and (18), we obtain

$$
\left|v\left(t_{2}\right)\right|_{L^{p+3 / q}}^{p+3 / q}+\int_{t_{1}}^{t_{2}}|v(s)|_{L^{p+3 / q}}^{p+3 / q} d s \leq\left|v\left(t_{1}\right)\right|_{L^{p+3 / q}}^{p+3 / q}+M \int_{t_{1}}^{t_{2}}|u(s)|_{L^{\infty}}^{2+p q} d s .
$$

Multiplying the above inequality by $p q /\left(2 C_{1} M(1+p q)\right)$ and adding the result to (19) yield (23) with

$$
C_{2}=\frac{1}{2 C_{1} M} \text { and } C_{3}=\frac{2 C_{1} M}{\min \{1, M\}}
$$

We next prove that the $L^{\infty}$-norms of $u$ and $v$ converge to zero as time increases to infinity.

LEMMA 5. There holds

$$
\begin{gathered}
\lim _{t \rightarrow+\infty}\left(|u(t)|_{L^{\infty}}+|v(s)|_{L^{\infty}}\right)=0, \\
\lim _{t \rightarrow+\infty}|u(t)|_{L^{1}}=M \text { and } \lim _{t \rightarrow+\infty}|v(t)|_{L^{1}}=0 .
\end{gathered}
$$

Proof. We first claim that the quasi-monotonicity of the right-hand side of (1)-(2) ensures that

$$
t \mapsto|u(t)|_{L^{\infty}}+|v(t)|_{L^{\infty}} \text { is nonincreasing. }
$$

Indeed consider $t_{0} \in[0,+\infty)$ and denote by $(X, Y)$ the solution to the system of ordinary differential equations

$$
\begin{gathered}
\frac{d X}{d t}=Y-X^{q}, \frac{d Y}{d t}=X^{q}-Y, \quad t \geq t_{0}, \\
X\left(t_{0}\right)=\left|u\left(t_{0}\right)\right|_{L^{\infty}}, Y\left(t_{0}\right)=\left|v\left(t_{0}\right)\right|_{L^{\infty}} .
\end{gathered}
$$

Then $(u-X, v-Y)$ satisfies

$$
\begin{aligned}
(u-X)_{t}+(u-X)_{x}-(u-X)_{x x} & =(v-Y)-\left(u^{q}-X^{q}\right), \\
(v-Y)_{t} & =\left(u^{q}-X^{q}\right)-(v-Y) .
\end{aligned}
$$

We put $\varrho(x)=\left(1+x^{2}\right)^{-1}$ for $x \in \mathbb{R}$. We multiply (30) by $\varrho \operatorname{sign}_{+}(u-X)$, (31) by $\varrho \operatorname{sign}_{+}(v-Y)$, add the resulting identities and integrate over $\left(t_{0}, t\right) \times \mathbb{R}$ to obtain, with 
the help of the Kato inequality,

$$
\begin{aligned}
\int(u & -X)_{+}(t) \varrho d x+\int(v-Y)_{+}(t) \varrho d x \\
\leq & \int_{t_{0}}^{t} \int(u-X)_{+}\left(\varrho_{x}+\varrho_{x x}\right) d x d s \\
& +\int_{t_{0}}^{t} \int\left((v-Y)_{+}-\left(u^{q}-X^{q}\right)_{+}\right) \varrho d x d s \\
& +\int_{t_{0}}^{t} \int\left(\left(u^{q}-X^{q}\right)_{+}-(v-Y)_{+}\right) \varrho d x d s \\
\leq & 3 \int(u-X)_{+}(t) \varrho d x .
\end{aligned}
$$

It then follows from the Gronwall lemma that

$$
\int(u-X)_{+}(t) \varrho d x+\int(v-Y)_{+}(t) \varrho d x=0
$$

for $t \geq t_{0}$; hence

$$
u(t, x) \leq X(t) \text { and } v(t, x) \leq Y(t)
$$

for $t \geq t_{0}$ and almost every $x \in \mathbb{R}$. Consequently, if $t \geq t_{0}$,

$$
|u(t)|_{L^{\infty}}+|v(t)|_{L^{\infty}} \leq X(t)+Y(t) .
$$

But the right-hand side of the previous inequality is obviously equal to $X\left(t_{0}\right)+Y\left(t_{0}\right)$ by (28)-(29) and the proof of the claim is complete.

As $t \mapsto|u(t)|_{L^{\infty}}+|v(t)|_{L^{\infty}}$ is bounded from below, it follows from (27) that there is $\ell \in[0,+\infty)$ such that

$$
\lim _{t \rightarrow+\infty}\left(|u(t)|_{L^{\infty}}+|v(t)|_{L^{\infty}}\right)=\ell .
$$

Now, on the one hand, we infer from (13) and the Hölder inequality that

$$
|v(t)|_{L^{\infty}} \leq\left|v_{0}\right|_{L^{\infty}} e^{-t}+\left(\int_{0}^{t} e^{s-t}|u(s)|_{L^{\infty}}^{2+q} d s\right)^{q /(2+q)}
$$

On the other hand, it follows from (19) with $p=1$ and $t_{1}=0$ that

$$
\int_{0}^{t}|u(s)|_{L^{\infty}}^{2+q} d s \leq 2 C_{1}\left(\left|u_{0}\right|_{L^{\infty}}^{q}\left|u_{0}\right|_{L^{1}}+\left|v_{0}\right|_{L^{\infty}}\left|u_{0}\right|_{L^{1}}\right)
$$

for each $t>0$, which entails that $t \mapsto|u(t)|_{L^{\infty}}^{2+q}$ belongs to $L^{1}(0,+\infty)$. It is then straightforward to check that

$$
\lim _{t \rightarrow+\infty} \int_{0}^{t} e^{s-t}|u(s)|_{L^{\infty}}^{2+q} d s=0 .
$$

Recalling (33) we conclude that

$$
\lim _{t \rightarrow+\infty}|v(t)|_{L^{\infty}}=0 .
$$

The above assertion and (32) next yield that $|u(t)|_{L^{\infty}}$ converges to $\ell$ as time increases to infinity. But $t \mapsto|u(t)|_{L^{\infty}}^{2+q}$ belongs to $L^{1}(0,+\infty)$ which implies $\ell=0$ and we have proved (25). 
In order to prove (26) we first notice that (13) yields

$$
|v(t)|_{L^{1}} \leq\left|v_{0}\right|_{L^{1}} e^{-t}+\int_{0}^{t} e^{s-t}|u(s)|_{L^{\infty}}^{q-1}|u(s)|_{L^{1}} d s .
$$

As $q>1$ it is easy to check that (18) and (25) ensure that the right-hand side of the above inequality converges to zero as time increases to infinity. Consequently,

$$
\lim _{t \rightarrow+\infty}|v(t)|_{L^{1}}=0
$$

and we use (14) to complete the proof of (26).

3. Temporal decay estimates. We keep the notations of Sec. 2 and assume further that

$$
u_{0, x}, v_{0, x} \in L^{1}(\mathbb{R}) \cap L^{\infty}(\mathbb{R}) .
$$

It follows from (18) and (27) that

$$
|u(t)|_{L^{\infty}}+|v(t)|_{L^{\infty}}+|u(t)|_{L^{1}}+|v(t)|_{L^{1}} \leq C_{0} \text { for } t \geq 0,
$$

where

$$
C_{0}=M+\left|u_{0}\right|_{L^{\infty}}+\left|v_{0}\right|_{L^{\infty}} .
$$

We now handle separately the cases $q \geq 3$ and $q \in[2,3)$. In the following, we denote by $C$ any positive constant which depends only on $q, u_{0}$, and $v_{0}$. The dependence of $C$ upon additional parameters will be indicated explicitly.

REMARK 4. Though the large time behaviour of solutions to (1)-(2) is the same for $q \geq 3$ and $q \in(2,3)$, the proof of Theorem 1 differs in the two cases. Though we have no clear explanation for this point, let us recall that $q=3$ is known to be a critical exponent for some one-dimensional parabolic equations. More precisely, $q=3$ is a critical exponent for the equation $u_{t}-u_{x x}+u^{q}=0$ with respect to the existence of solutions with measures as initial data $[\mathrm{BF}]$ and to the large time behaviour; see, e.g., [Va] and the references therein. Also, the second term in the asymptotic development of solutions of the scalar convection-diffusion equation $u_{t}+\left(u^{q}\right)_{x}-u_{x x}=0$ differs when $q>3$ and when $q \in(2,3)$ $[\mathrm{Zu}]$.

3.1. The case $q \geq 3$.

Lemma 6. For each $p \in[1, \infty)$ and $t \in(0,+\infty)$ there holds

$$
t^{(1-1 / p) / 2}|u(t)|_{L^{p}}+t^{(q-1 / p) / 2}|v(t)|_{L^{p}} \leq C(p) .
$$

Proof. Consider $p \geq 1$. Then $p+1 \geq p+3 / q$ and it follows from (35) that

$$
|v(t)|_{L^{1+p}}^{1+p} \leq C_{0}^{(q-3) / q}|v(t)|_{L^{p+3 / q}}^{p+3 / q}, \quad t \geq 0 .
$$

We now estimate $D_{p}$ defined by (24) from below by a power of $E_{p}$ defined by (22). More precisely it follows from (37) and (35) that

$$
\begin{aligned}
E_{p} & \leq|u|_{L^{1}}|u|_{L^{\infty}}^{p q}+\left(q C_{0}^{(q-3) / q}+C_{2}\right)|v|_{L^{p+3 / q}}^{p+3 / q} \\
& \leq C D_{p}^{(p q) /(2+p q)}+C|v|_{L^{p+3 / q}}^{(p+3 / q)(p q) /(2+p q)} \\
E_{p} & \leq C D_{p}^{(p q) /(2+p q)} .
\end{aligned}
$$


We then infer from (38) and (23) that

$$
\int_{t_{1}}^{t_{2}} E_{p}(s)^{(2+p q) / p q} d s \leq C(p) E_{p}\left(t_{1}\right), \quad 0 \leq t_{1} \leq t_{2} .
$$

Since $E_{p}$ is a nonincreasing function on $(0,+\infty)$, the above inequality and [Ko, Theorem 9.1] yield

$$
E_{p}(t) \leq C(p) t^{-(p q) / 2}, \quad t>0
$$

Therefore

$$
|u(t)|_{L^{1+p q}} \leq C(p) t^{-(1-1 /(1+p q)) / 2}, \quad t>0,
$$

which gives (36) for $u$ and $p \geq 1+q$. The assertion (36) for $u$ and $p \in[1,1+q$ ) then follows by interpolation with $(35)$.

Consider next $p \geq 0$ and $t \in(0,+\infty)$. We infer from (13) and (35) that

$$
\begin{aligned}
|v(t)|_{L^{1+p}} & \leq|v(t / 2)|_{L^{1+p}} e^{-t / 2}+\int_{t / 2}^{t} e^{s-t}|u(s)|_{L^{q(p+1)}}^{q} d s \\
|v(t)|_{L^{1+p}} & \leq C e^{-t / 2}+\sup _{s \in[t / 2, t]}|u(s)|_{L^{q(p+1)}}^{q} .
\end{aligned}
$$

We now use (36) for $u$ in the above inequality to deduce (36) for $v$.

The constant $C(p)$ obtained in Lemma 6 blows up as $p$ increases to infinity. Thus, we cannot obtain $L^{\infty}$-decay estimates for $u$ and $v$ by letting $p \rightarrow+\infty$ in (36). We rather make use of Lemma 6 together with the regularizing properties of the semigroup $\widetilde{G}(t)$ to obtain temporal decay estimates in $L^{\infty}(\mathbb{R})$ and in $H^{1}(\mathbb{R})$.

Lemma 7. For $t \in(0,+\infty)$ there holds

$$
\begin{gathered}
t^{1 / 2}|u(t)|_{L^{\infty}}+t^{q / 2}|v(t)|_{L^{\infty}} \leq C, \\
t^{3 / 4}\left(\left|u_{x}(t)\right|_{L^{2}}+\left|v_{x}(t)\right|_{L^{2}}\right) \leq C .
\end{gathered}
$$

Proof. Let $t \in(0,+\infty)$. The Duhamel formula (12) yields

$$
\begin{aligned}
|u(2 t)|_{L^{\infty}} \leq & |\tilde{G}(t) u(t)|_{L^{\infty}}+\int_{0}^{t}\left|\widetilde{G}(t-s)\left(v-u^{q}\right)(s+t)\right|_{L^{\infty}} d s \\
\leq & C|u(t)|_{L^{1}} t^{-1 / 2} \\
& +C \int_{0}^{t}(t-s)^{-1 / 2}\left(|v(s+t)|_{L^{1}}+|u(s+t)|_{L^{q}}^{q}\right) d s .
\end{aligned}
$$

Owing to (35) and (36) we further obtain

$$
|u(2 t)|_{L^{\infty}} \leq C t^{-1 / 2}\left(1+t^{(3-q) / 2}\right) .
$$

As $q \geq 3$ we conclude that

$$
|u(t)|_{L^{\infty}} \leq C t^{-1 / 2} \text { for } t \geq 1 .
$$

As $|u(t)|_{L^{\infty}} \leq C_{0}$ by (35) we finally obtain

$$
t^{1 / 2}|u(t)|_{L^{\infty}} \leq C, \quad t \in(0,+\infty)
$$


We next infer from (13) and (35) that

$$
|v(2 t)|_{L^{\infty}} \leq C_{0} e^{-t}+\sup _{s \in[t, 2 t]}|u(s)|_{L^{\infty}}^{q}
$$

which easily yields (39) by (41).

We next claim that

$$
\left(\left|u_{x}(t)\right|_{L^{2}}+\left|v_{x}(t)\right|_{L^{2}}\right) \leq C, \quad t \in[0,1] .
$$

Indeed, putting $\xi=u_{x}$ and $\eta=v_{x}$, we infer from (1)-(2) that

$$
\begin{aligned}
\xi_{t}+\xi_{x}-\xi_{x x} & =\eta-q u^{q-1} \xi, \\
\eta_{t} & =q u^{q-1} \xi-\eta .
\end{aligned}
$$

By (35) we have

$$
\frac{d}{d t} \int\left(|\xi|^{2}+|\eta|^{2}\right) d x \leq \int\left(|\eta \xi|+q \xi^{2}-\eta^{2}+q|\eta \xi|\right) d x
$$

for $t \in[0,1]$, from which the claim (42) follows by the Gronwall lemma.

We next use again the Duhamel formula (12) to obtain

$$
\begin{aligned}
\left|u_{x}(2 t)\right|_{L^{2}} \leq & \left|\widetilde{G}_{x}(t) u(t)\right|_{L^{2}}+\int_{0}^{t}\left|\widetilde{G}_{x}(t-s)\left(v-u^{q}\right)(s+t)\right|_{L^{2}} d s \\
\leq & C|u(t)|_{L^{1}} t^{-3 / 4} \\
& +C \int_{0}^{t}(t-s)^{-3 / 4}\left(|v(s+t)|_{L^{1}}+|u(s+t)|_{L^{q}}^{q}\right) d s .
\end{aligned}
$$

The above inequality, (35) and (36) next entail

$$
\left|u_{x}(2 t)\right|_{L^{2}} \leq C t^{-3 / 4}\left(1+t^{(3-q) / 2}\right),
$$

and (40) for $u$ follows directly from (42) and the above inequality. We next proceed as before to prove that (40) for $u,(35)$ and (13) imply that (40) for $v$ also holds true.

3.2. The case $q \in[2,3)$. We first show that the estimate (36) still holds true, its proof being slightly different.

LEMma 8. For each $p \in[1, \infty)$ and $t \in(0,+\infty)$, there holds

$$
t^{(1-1 / p) / 2}|u(t)|_{L^{p}}+t^{(q-1 / p) / 2}|v(t)|_{L^{p}} \leq C(p) .
$$

Proof. Consider $p \geq 1$. Then $p+1 \leq p+3 / q$ and (35) and the Hölder inequality yield

$$
|v(t)|_{L^{1+p}}^{1+p} \leq C(p)|v(t)|_{L^{p+3 / q}}^{(p+3 / q)(p q) /(p q+3-q)}, \quad t \geq 0 .
$$

Owing to (35) and (44), we may estimate $E_{p}$ from above as follows.

$$
\begin{aligned}
E_{p} \leq & |u|_{L^{1}}|u|_{L^{\infty}}^{p q}+C(p)|v|_{L^{p+3 / q}}^{(p+3 / q)(p q) /(p q+3-q)} \\
& +C_{2}|v|_{L^{p+3 / q}}^{p+3 / q} \\
\leq & C D_{p}^{(p q) /(2+p q)}+C(p)|v|_{L^{p+3 / q}}^{(p+3 / q)(p q) /(2+p q)},
\end{aligned}
$$

since $p q+3-q<p q+2$. Consequently,

$$
E_{p} \leq C(p) D_{p}^{(p q) /(2+p q)},
$$


and we argue as in the proof of Lemma 6 to conclude that (43) holds true.

REMARK 5. Lemma 8 is actually valid for $q \in(1,3)$. Moreover it is clear from the proofs of Lemma 6 and Lemma 8 that the constants $C(p)$ in (36) and (43) only depend on $q, M,\left|u_{0}\right|_{L^{\infty}},\left|v_{0}\right|_{L^{\infty}}$, and $p$.

The proof of Lemma 7 relies heavily on the assumption $q \geq 3$ and clearly does not extend to the present case. Still, we have the following (weaker) result.

Lemma 9. For $t \in(0,+\infty)$, there holds

$$
t^{1 / 2}|u(t)|_{L^{\infty}}+t^{q / 2}|v(t)|_{L^{\infty}} \leq C .
$$

In addition, for each $\tau \in(0,+\infty)$ and $t \in(\tau,+\infty)$, we have

$$
t^{1 / 2}|(u+v)(t)|_{H^{1 / 2}} \leq C(\tau) .
$$

Proof. The proof is divided into three steps.

Step 1. We first claim that, if $p \in[1, \infty)$ and $t \in(0,+\infty)$, there holds

$$
\left|u_{x}(t)\right|_{L^{p}} \leq C(p) t^{(1-1 / p) / 2}\left(t^{-1 / 2}+t^{(2-q) / 2}\right) .
$$

Indeed, the Duhamel formula (12) yields

$$
\begin{aligned}
\left|u_{x}(2 t)\right|_{L^{p}} \leq & \left|\widetilde{G}_{x}(t) u(t)\right|_{L^{p}}+\int_{0}^{t}\left|\widetilde{G}_{x}(t-s)\left(v-u^{q}\right)(s+t)\right|_{L^{p}} d s \\
\leq & C|u(t)|_{L^{1}} t^{-(2-1 / p) / 2} \\
& +C \int_{0}^{t}(t-s)^{-(2-1 / p) / 2}\left(|v(s+t)|_{L^{1}}+|u(s+t)|_{L^{q}}^{q}\right) d s
\end{aligned}
$$

and (47) follows at once from (35), (43), and the above inequality.

Step 2. We next deduce from (47) some temporal decay estimates for $v_{x}$. More precisely, if $p \in[1, \infty)$ and $t \in(0,+\infty)$, we infer from (13), the Hölder inequality, (34), (35), (43), and (47) that

$$
\begin{aligned}
\left|v_{x}(t)\right|_{L^{p}} \leq & e^{-t}\left|v_{0, x}\right|_{L^{p}}+q \int_{0}^{t / 2} e^{s-t}|u(s)|_{L^{\infty}}^{q-1}\left|u_{x}(s)\right|_{L^{p}} d s \\
& +q \int_{t / 2}^{t} e^{s-t}|u(s)|_{L^{2 p(q-1)}}^{q-1}\left|u_{x}(s)\right|_{L^{2 p}} d s \\
\leq & C e^{-t}+C(p) e^{-t / 2} t^{1 / 2 p}\left(1+t^{(3-q) / 2}\right) \\
& +C(p) \int_{t / 2}^{t} e^{s-t} s^{(q-1 / p) / 2}\left(s^{-1 / 2}+s^{(2-q) / 2}\right) d s \\
\leq & C(p)\left(e^{-t / 4}+t^{(q-1 / p) / 2}\left(t^{-1 / 2}+t^{(2-q) / 2}\right)\right) . \\
\left|v_{x}(t)\right|_{L^{p}} \leq & C(p) t^{(q-1 / p) / 2}\left(1+t^{-1 / 2}+t^{(2-q) / 2}\right), \quad t>0 .
\end{aligned}
$$

Step 3. We put $w=u+v$. By (1)-(2), $w$ is the solution to

$$
w_{t}+w_{x}-w_{x x}=v_{x}-v_{x x} \text { in }(0,+\infty) \times \mathbb{R}
$$

with initial datum $w(0)=u_{0}+v_{0}$. We infer from the Duhamel formula that

$$
w(2 t)=\widetilde{G}(t) w(t)+\int_{0}^{t} \widetilde{G}_{x}(t-s)\left(v-v_{x}\right)(s+t) d s, \quad t \geq 0 .
$$


It first follows from (35), (43), (48), and (49) that

$$
\begin{aligned}
|w(2 t)|_{L^{\infty}} \leq & C t^{-1 / 2}|w(t)|_{L^{1}} \\
& +C \int_{0}^{t}(t-s)^{-3 / 4}\left(|v(s+t)|_{L^{2}}+\left|v_{x}(s+t)\right|_{L^{2}}\right) d s \\
\leq & C t^{-1 / 2}+C t^{-(q-1) / 2}\left(1+t^{-1 / 2}+t^{(2-q) / 2}\right) \\
\leq & C t^{-1 / 2}\left(1+t^{-(q-2) / 2}\left(1+t^{-1 / 2}+t^{(2-q) / 2}\right)\right) .
\end{aligned}
$$

Since $q \geq 2$, we obtain

$$
|w(t)|_{L^{\infty}} \leq C t^{-1 / 2} \text { for } t \geq 1
$$

Recalling (35), the above inequality and the nonnegativity of $u$ and $v$ yield

$$
|u(t)|_{L^{\infty}} \leq C t^{-1 / 2} \text { for } t \geq 0 .
$$

We now argue as in the proof of Lemma 7 to show that (45) follows from the previous estimate.

Using again the Duhamel formula (49), we obtain

$$
\begin{aligned}
|w(2 t)|_{H^{1 / 2}} \leq & C t^{-1 / 2}|w(t)|_{L^{1}} \\
& +C \int_{0}^{t}(t-s)^{-3 / 4}\left(|v(s+t)|_{L^{2}}+\left|v_{x}(s+t)\right|_{L^{2}}\right) d s .
\end{aligned}
$$

Proceeding as above, we deduce from (35), (43), and (48) that

$$
|w(2 t)|_{H^{1 / 2}} \leq C t^{-1 / 2}\left(1+t^{-(q-2) / 2}\left(1+t^{-1 / 2}+t^{(2-q) / 2}\right)\right),
$$

hence (46) since $q \geq 2$.

4. Proof of Theorem 1. We now proceed with the proof of Theorem 1. Let $\left(u_{0}, v_{0}\right)$ be two measurable functions satisfying (4) and (34). We put $(u, v)(t)=S_{t}\left(u_{0}, v_{0}\right)$ for $t \geq 0$ and

$$
M:=\int\left(u_{0}+v_{0}\right)(x) d x .
$$

Following the analysis of $[\mathrm{GvDD}]$, we introduce the new unknown functions $(U, V)$ defined by

$$
(U, V)(t, x)=(u, v)(t, x+t) \text { for }(t, x) \in(0,+\infty) \times \mathbb{R} .
$$

Then $(U, V)$ solves

$$
\begin{gathered}
U_{t}-U_{x x}=V-U^{q} \text { in }(0,+\infty) \times \mathbb{R}, \\
V_{t}-V_{x}=U^{q}-V \text { in }(0,+\infty) \times \mathbb{R},
\end{gathered}
$$

with initial data $(U, V)(0)=\left(u_{0}, v_{0}\right)$. We next define

$$
U_{\lambda}(t, x)=\lambda U\left(\lambda^{2} t, \lambda x\right) \text { and } V_{\lambda}(t, x)=\lambda^{q} V\left(\lambda^{2} t, \lambda x\right)
$$

for $(t, x) \in(0,+\infty) \times \mathbb{R}$ and $\lambda \geq 1$. We also put

$$
W_{\lambda}(t, x)=\lambda(U+V)\left(\lambda^{2} t, \lambda x\right)=U_{\lambda}(t, x)+\lambda^{1-q} V_{\lambda}(t, x) .
$$


It follows from $(51)-(52)$ that $\left(U_{\lambda}, V_{\lambda}\right)$ solves

$$
\begin{aligned}
U_{\lambda, t}-U_{\lambda, x x} & =\lambda^{2-q} V_{\lambda, x}-\lambda^{1-q} V_{\lambda, t} \text { in }(0,+\infty) \times \mathbb{R}, \\
V_{\lambda}-U_{\lambda}^{q} & =\lambda^{-1} V_{\lambda, x}-\lambda^{-2} V_{\lambda, t} \text { in }(0,+\infty) \times \mathbb{R} .
\end{aligned}
$$

Observe that an equivalent formulation of (55) is

$$
W_{\lambda, t}-U_{\lambda, x x}=\lambda^{2-q} V_{\lambda, x} \text { in }(0,+\infty) \times \mathbb{R}
$$

We now make use of the analysis of the previous section to derive estimates on $\left(U_{\lambda}, V_{\lambda}, W_{\lambda}\right)$ which are uniform with respect to $\lambda \geq 1$. In the following, we denote by $C$ any positive constant which only depends on $q, M, u_{0}$, and $v_{0}$. The dependence of $C$ upon additional parameters will be indicated explicitly.

Lemma 10. Consider $p \in[1, \infty]$ and $t \in(0,+\infty)$. There holds

$$
\begin{aligned}
t^{(1-1 / p) / 2}\left|U_{\lambda}(t)\right|_{L^{p}} & +t^{(q-1 / p) / 2}\left|V_{\lambda}(t)\right|_{L^{p}} \\
& +\frac{t^{(1-1 / p) / 2}}{1+t^{(1-q) / 2}}\left|W_{\lambda}(t)\right|_{L^{p}} \leq C(p) .
\end{aligned}
$$

In addition, if $q \geq 3$, we have

$$
t^{3 / 4}\left|W_{\lambda, x}(t)\right|_{L^{2}} \leq C \text { for } t \geq 0,
$$

while for $q \in[2,3)$ and $\tau>0$, there holds

$$
t^{1 / 2}\left|W_{\lambda}(t)\right|_{H^{1 / 2}} \leq C(\tau) \text { for } t \geq \tau \text {. }
$$

Proof. Let us first consider $p \in[1, \infty]$ and $t \in(0,+\infty)$. By $(50)$, we have

$$
|U(t)|_{L^{p}}=|u(t)|_{L^{p}} \text { and }|V(t)|_{L^{p}}=|v(t)|_{L^{p}} .
$$

The assertion (58) then follows from (36), (39), (43), and (45) by elementary computations. Assuming next that $q \geq 3,(59)$ is a straightforward consequence of $(40)$. We finally consider $q \in[2,3)$. Let $t \in(0,+\infty)$. As $\lambda \geq 1$ we have

$$
\left|W_{\lambda}(t)\right|_{H^{1 / 2}} \leq \lambda\left|W_{\lambda}\left(\lambda^{2} t\right)\right|_{H^{1 / 2}} .
$$

For $\tau>0, \lambda \geq 1$, and $t \in(\tau,+\infty)$, we have $\lambda^{2} t \geq \tau$ and we infer from (46) that (60) holds true.

Lemma 11. For $t_{1} \in(0,+\infty)$ and $t_{2} \in\left(t_{1},+\infty\right)$, there holds

$$
\left|W_{\lambda, t}\right|_{L^{2}\left(t_{1}, t_{2} ; H^{-1}\right)} \leq C\left(t_{1}, t_{2}\right) .
$$

Proof. Consider $t_{1} \in(0,+\infty), t_{2} \in\left(t_{1},+\infty\right)$, and $\varphi \in L^{2}\left(t_{1}, t_{2} ; H^{1}(\mathbb{R})\right)$. We first infer from (20) (with $p=1 / q$ ) that

$$
\int_{\lambda^{2} t_{1}}^{\lambda^{2} t_{2}} \int\left|u_{x}(s, x)\right|^{2} d x d s \leq C\left(\left|u\left(\lambda^{2} t_{1}\right)\right|_{L^{2}}^{2}+\left|v\left(\lambda^{2} t_{1}\right)\right|_{L^{(1+q) / q}}^{(1+q) / q}\right) .
$$

Consequently

$$
\begin{aligned}
\int_{t_{1}}^{t_{2}} \int\left|U_{\lambda, x}(s, x)\right|^{2} d x d s & \leq \lambda \int_{\lambda^{2} t_{1}}^{\lambda^{2} t_{2}} \int\left|u_{x}(s, x)\right|^{2} d x d s \\
& \leq C \lambda\left(\left|u\left(\lambda^{2} t_{1}\right)\right|_{L^{2}}^{2}+\left|v\left(\lambda^{2} t_{1}\right)\right|_{L^{(1+q) / q}}^{(1+q) / q}\right)
\end{aligned}
$$


and Lemma 6 and Lemma 8 entail

$$
\begin{aligned}
\int_{t_{1}}^{t_{2}} \int\left|U_{\lambda, x}(s, x)\right|^{2} d x d s & \leq C\left(t_{1}^{-1 / 2}+\lambda^{1-q} t_{1}^{-q / 2}\right) \\
& \leq C\left(t_{1}\right)
\end{aligned}
$$

(recall that $\lambda \geq 1$ and $q \geq 2$ ). We next infer from (57), (58), and (62) that

$$
\begin{aligned}
& \left|\int_{t_{1}}^{t_{2}} \int W_{\lambda, t} \varphi d x d s\right| \\
& \quad \leq\left(\left|U_{\lambda, x}\right|_{L^{2}\left(t_{1}, t_{2} ; L^{2}\right)}+\lambda^{2-q}\left|V_{\lambda}\right|_{L^{2}\left(t_{1}, t_{2} ; L^{2}\right)}\right)\left|\varphi_{x}\right|_{L^{2}\left(t_{1}, t_{2} ; L^{2}\right)} \\
& \quad \leq C\left(t_{1}, t_{2}\right)|\varphi|_{L^{2}\left(t_{1}, t_{2} ; H^{1}\right)},
\end{aligned}
$$

from which (61) follows.

Lemma 12. For $T \in(0,+\infty)$, there holds

$$
\lim _{R \rightarrow+\infty} \sup _{\lambda \geq 1, t \in[0, T]} \int_{\{|x| \geq R\}} W_{\lambda}(t, x) d x=0 .
$$

Proof. Let $\vartheta$ be a smooth function in $\mathcal{C}^{\infty}(\mathbb{R})$ satisfying $0 \leq \vartheta \leq 1$ and

$$
\vartheta(x)=0 \text { if }|x| \leq 1 / 2 \text { and } \vartheta(x)=1 \text { if }|x| \geq 1 .
$$

For $R \geq 1$ and $x \in \mathbb{R}$, we put $\vartheta_{R}(x)=\vartheta(x / R)$. Let $t \in[0, T]$. It follows from (57) that

$$
\begin{aligned}
\int \vartheta_{R}(x) W_{\lambda}(t, x) d x \leq & \int\left(u_{0}+v_{0}\right)(x) \vartheta_{R}(x / \lambda) d x \\
& +\frac{\left|\vartheta_{x x}\right|_{L^{\infty}}}{R^{2}} \int_{0}^{t}\left|U_{\lambda}(s)\right|_{L^{1}} d s \\
& +\frac{\lambda^{2-q}\left|\vartheta_{x}\right|_{L^{\infty}}}{R} \int_{0}^{t}\left|V_{\lambda}(s)\right|_{L^{1}} d s
\end{aligned}
$$

On the one hand, it follows from (58) that

$$
\int_{0}^{t}\left|U_{\lambda}(s)\right|_{L^{1}} d s \leq C T .
$$

On the other hand, using Lemma 6 and Lemma 8, we obtain

$$
\begin{aligned}
& \lambda^{2-q} \int_{0}^{t}\left|V_{\lambda}(s)\right|_{L^{1}} d s=\lambda \int_{0}^{t}\left|V\left(\lambda^{2} s\right)\right|_{L^{1}} d s \\
& \leq \lambda \int_{0}^{T / \lambda^{2}} M d s+C \lambda^{2-q} \int_{T / \lambda^{2}}^{T} s^{-(q-1) / 2} d s \\
& \leq C(T)+C \begin{cases}\lambda^{2-q} T^{(3-q) / 2} & \text { if } q \in[2,3), \\
\lambda^{-1} \ln \lambda & \text { if } q=3, \\
\lambda^{-1} T^{(3-q) / 2} & \text { if } q>3\end{cases} \\
& \leq C(T) \text {. }
\end{aligned}
$$


Combining the above estimates yields, since $\lambda \geq 1$,

$$
\int \vartheta_{R}(x) W_{\lambda}(t, x) d x \leq \int_{\{|x| \geq R\}}\left(u_{0}+v_{0}\right)(x) d x+\frac{C(T, \vartheta)}{R} .
$$

As $u_{0}+v_{0}$ belongs to $L^{1}(\mathbb{R})$, Lemma 12 follows at once from the above inequality.

Summarizing the outcome of Lemma 10 and Lemma 11, we have proved that $\left(W_{\lambda}\right)_{\lambda \geq 1}$ is bounded in $L^{\infty}\left(t_{1}, t_{2} ; H^{s}(\mathbb{R})\right)$ and in $W^{1,2}\left(t_{1}, t_{2} ; H^{-1}(\mathbb{R})\right)$ for every $t_{1}>0$ and $t_{2}>t_{1}$, the exponent $s$ being equal to 1 if $q \geq 3$ and to $1 / 2$ if $q \in[2,3)$. Owing to the compactness of the embedding of $H_{\mathrm{loc}}^{s}(\mathbb{R})$ in $L_{\mathrm{loc}}^{2}(\mathbb{R})$, we infer from [Si, Corollary 4] that $\left(W_{\lambda}\right)$ is relatively compact in $\mathcal{C}\left(\left[t_{1}, t_{2}\right] ; L_{\text {loc }}^{2}(\mathbb{R})\right)$ for every $t_{1}>0$ and $t_{2}>t_{1}$. Furthermore Lemma 12 provides the uniform integrability of $\left(W_{\lambda}\right)$ for large values of $x$ and we finally conclude that

$$
\begin{aligned}
& \left(W_{\lambda}\right) \text { is relatively compact in } \mathcal{C}\left(\left[t_{1}, t_{2}\right] ; L^{1}(\mathbb{R})\right) \text { for every } \\
& t_{1}>0 \text { and } t_{2}>t_{1} .
\end{aligned}
$$

Therefore there is a sequence $\left(\lambda_{n}\right), \lambda_{n} \rightarrow+\infty$, and a function

$$
U_{\infty} \in \mathcal{C}\left((0,+\infty) ; L^{1}(\mathbb{R})\right)
$$

such that

$$
\lim _{n \rightarrow+\infty} \sup _{t \in\left[t_{1}, t_{2}\right]}\left|W_{\lambda_{n}}(t)-U_{\infty}(t)\right|_{L^{1}}=0
$$

for every $t_{1}>0$ and $t_{2}>t_{1}$. Notice first that (64) and the nonnegativity of $W_{\lambda}$ ensure that

$$
U_{\infty}(t) \geq 0 \text { a.e. in } \mathbb{R} \text { for } t>0 .
$$

Since $q \geq 2$, we next infer from (58) that

$$
\lim _{\lambda \rightarrow+\infty} \sup _{t \in\left[t_{1}, t_{2}\right]} \lambda^{1-q}\left|V_{\lambda}(t)\right|_{L^{1}}=0
$$

for every $t_{1}>0$ and $t_{2}>t_{1}$, which yields, together with (64),

$$
\lim _{n \rightarrow+\infty} \sup _{t \in\left[t_{1}, t_{2}\right]}\left|U_{\lambda_{n}}(t)-U_{\infty}(t)\right|_{L^{1}}=0 .
$$

We now identify the behaviour of $U_{\infty}(t)$ as $t \rightarrow 0$. Consider $\varphi \in \mathcal{D}(\mathbb{R}), t \in(0,1)$, and $s \in(0, t)$. It follows from $(57)$ that

$$
\begin{aligned}
& \left|\int W_{\lambda_{n}}(t, x) \varphi(x) d x-\int W_{\lambda_{n}}(s, x) \varphi(x) d x\right| \\
& \quad \leq C(\varphi) \int_{s}^{t}\left(\left|U_{\lambda_{n}}(\sigma)\right|_{L^{1}}+\lambda_{n}^{2-q}\left|V_{\lambda_{n}}(\sigma)\right|_{L^{1}}\right) d \sigma .
\end{aligned}
$$

Thanks to (58), we further obtain

$$
\begin{gathered}
\left|\int W_{\lambda_{n}}(t, x) \varphi(x) d x-\int W_{\lambda_{n}}(s, x) \varphi(x) d x\right| \\
\leq C(\varphi)\left(t+\lambda_{n}^{2-q} \int_{s}^{t} \sigma^{-(q-1) / 2} d \sigma\right) .
\end{gathered}
$$


We first treat the case $q \in[2,3)$. On the one hand, $(q-1) / 2<1$ and we may let $s \rightarrow 0$ in (68) to obtain

$$
\left|\int W_{\lambda_{n}}(t, x) \varphi(x) d x-\int W_{\lambda_{n}}(0, x) \varphi(x) d x\right| \leq C(\varphi) \quad\left(t+t^{(3-q) / 2}\right) .
$$

On the other hand, it readily follows from (53) that

$$
\lim _{\lambda \rightarrow+\infty} \int W_{\lambda}(0, x) \varphi(x) d x=M \varphi(0) .
$$

Recalling (64) we may pass to the limit as $n \rightarrow+\infty$ in the previous inequality and obtain

$$
\left|\int U_{\infty}(t, x) \varphi(x) d x-M \varphi(0)\right| \leq C(\varphi) \quad\left(t+t^{(3-q) / 2}\right) .
$$

We then let $t \rightarrow 0$ and conclude that

$$
\lim _{t \rightarrow 0} \int U_{\infty}(t, x) \varphi(x) d x=M \varphi(0) .
$$

We next handle the case $q \geq 3$. We fix $\alpha \in(2,2(q-2) /(q-3))$ and take $s=\lambda_{n}^{-\alpha}$ in (68). We thus obtain

$$
\begin{gathered}
\left|\int W_{\lambda_{n}}(t, x) \varphi(x) d x-\int W_{\lambda_{n}}\left(\lambda_{n}^{-\alpha}, x\right) \varphi(x) d x\right| \\
\leq C(\varphi) \begin{cases}\left(t+\lambda_{n}^{2-q-\alpha(3-q) / 2}\right) & \text { if } q>3, \\
\left(t+\lambda_{n}^{-1} \ln \left(\lambda_{n}^{-\alpha} t\right)\right) & \text { if } q=3 .\end{cases}
\end{gathered}
$$

Owing to the choice of $\alpha$ we may let $n \rightarrow+\infty$ in the above inequality and use (64) to conclude that

$$
\limsup _{n \rightarrow+\infty}\left|\int U_{\infty}(t, x) \varphi(x) d x-\int W_{\lambda_{n}}\left(\lambda_{n}^{-\alpha}, x\right) \varphi(x) d x\right| \leq C(\varphi) t .
$$

We next infer from (54) that

$$
\begin{aligned}
& \left|\int W_{\lambda_{n}}(0, x) \varphi(x) d x-\int W_{\lambda_{n}}\left(\lambda_{n}^{-\alpha}, x\right) \varphi(x) d x\right| \\
& \leq C(\varphi)\left|W\left(\lambda_{n}^{2-\alpha}\right)-W(0)\right|_{L^{1}} .
\end{aligned}
$$

Since $\alpha>2$ and $W \in \mathcal{C}\left([0,+\infty) ; L^{1}(\mathbb{R})\right)$, we finally obtain

$$
\lim _{n \rightarrow+\infty} \int W_{\lambda_{n}}(0, x) \varphi(x) d x-\int W_{\lambda_{n}}\left(\lambda_{n}^{-\alpha}, x\right) \varphi(x) d x=0 .
$$

Combining (71), (72), and (69) then yields that (70) also holds for $q \geq 3$. A standard density argument and Lemma 12 further warrant that (70) holds true for every $\varphi \in$ $\mathcal{B C}(\mathbb{R})$, that is,

$$
\lim _{t \rightarrow 0} \int U_{\infty}(t, x) \varphi(x) d x=M \varphi(0) \text { for } \varphi \in \mathcal{B C}(\mathbb{R}) .
$$

Here $\mathcal{B C}(\mathbb{R})$ denotes the space of bounded and continuous functions on $\mathbb{R}$. 
We finally identify the equation satisfied by $U_{\infty}$. We consider $\varphi \in \mathcal{D}((0,+\infty) \times \mathbb{R})$ and infer from (56)-(57) that

$$
\begin{gathered}
\int_{0}^{+\infty} \int\left(W_{\lambda_{n}} \varphi_{t}+U_{\lambda_{n}} \varphi_{x x}\right) d x d s=\lambda_{n}^{2-q} \int_{0}^{+\infty} \int V_{\lambda_{n}} \varphi_{x} d x d s \\
\int_{0}^{+\infty} \int\left(V_{\lambda_{n}}-U_{\lambda_{n}}^{q}\right) \varphi d x d s=\int_{0}^{+\infty} \int\left(\lambda_{n}^{-2} \varphi_{t}-\lambda_{n}^{-1} \varphi_{x}\right) V_{\lambda_{n}} d x d s
\end{gathered}
$$

Since $\left(V_{\lambda_{n}}\right)$ is bounded in $L_{\text {loc }}^{1}\left((0,+\infty) ; L^{1}(\mathbb{R})\right)$ by $(58),(58)$ and $(67)$ allows to pass to the limit as $n \rightarrow+\infty$ in (75) to obtain that

$$
\left(V_{\lambda_{n}}\right) \text { converges to } U_{\infty}^{q} \text { in } \mathcal{D}^{\prime}((0,+\infty) \times \mathbb{R}) \text {. }
$$

We may then pass to the limit as $n \rightarrow+\infty$ in (74) and use (64), (67), and (76) to conclude that $U_{\infty}$ is a solution in $\mathcal{D}^{\prime}((0,+\infty) \times \mathbb{R})$ to

$$
U_{\infty, t}-U_{\infty, x x}=0 \text { if } q>2
$$

and

$$
U_{\infty, t}-U_{\infty, x x}=\left(U_{\infty}^{2}\right)_{x} \text { if } q=2 .
$$

In addition, it follows from (58) and (67) that

$$
\sup _{t \in(0,+\infty)}\left(\left|U_{\infty}(t)\right|_{L^{1}}+t^{1 / 2}\left|U_{\infty}(t)\right|_{L^{\infty}}\right)<\infty .
$$

We have thus proved that $U_{\infty}$ is a nonnegative solution to either (77) or (78) with initial datum $M \delta$ (recall (73)). Such a solution being unique (see [EVZ] for (78)) we conclude that $U_{\infty}$ is the fundamental solution to the linear heat equation (77) with mass $M$ if $q>2$ and the source-type solution to the viscous Burgers equation (78) with mass $M$ if $q=2$. Therefore $U_{\infty}=S_{M}$ with the notation in Theorem 1. Furthermore, the previous arguments guarantee that $\left(W_{\lambda}\right)$ has only one cluster point $S_{M}$ as $\lambda \rightarrow+\infty$. The relative compactness of $\left(W_{\lambda}\right)$ then entails that the whole family $\left(W_{\lambda}\right)$ converges to $S_{M}$ in $\mathcal{C}\left(\left[t_{1}, t_{2}\right] ; L^{1}(\mathbb{R})\right)$ as $\lambda \rightarrow+\infty$ for every $t_{1}>0$ and $t_{2}>t_{1}$. Recalling (66), so does $\left(U_{\lambda}\right)$; that is,

$$
\lim _{\lambda \rightarrow+\infty}\left|U_{\lambda}(t)-S_{M}(t)\right|_{L^{1}}=0 \text { for } t \in(0,+\infty) .
$$

As $S_{M}$ has a self-similar form, (80) may also be written as

$$
\lim _{t \rightarrow+\infty}\left|U(t)-S_{M}(t)\right|_{L^{1}}=0
$$

hence (5) for $p=1$. As $t \mapsto t^{1 / 2}|U(t)|_{L^{\infty}}$ is bounded by (39) and (45), the assertion (5) for $p \in(1, \infty)$ easily follows by interpolation and the proof of Theorem 1 is complete for initial data enjoying the additional regularity (34). The case of initial data satisfying only (4) then follows by a density argument combined with the contraction property (16), keeping in mind Remark 5.

Acknowledgements. This work was supported by TMR contract HCL \# ERBFMRXCT960033 and was performed while the second author enjoyed the hospitality of the Departamento de Matemáticas, UPV/EHU. The first author was also supported by DGES grant PB96-0663. 


\section{REFERENCES}

[BF] H. Brezis and A. Friedman, Nonlinear parabolic equations involving measure as initial conditions, J. Math. Pures Appl. 62 (1983), 73-97.

[C] I.-L. Chern, Long-time effect of relaxation for hyperbolic conservation laws, Comm. Math. Phys. 172 (1995), 39-55.

[CT] M. G. Crandall and L. Tartar, Some relations between nonexpansive and order preserving mappings, Proc. Amer. Math. Soc. 78 (1980), 385-390.

[EVZ] M. Escobedo, J. L. Vazquez and E. Zuazua, Asymptotic behaviour and source-type solutions for a diffusion-convection equation, Arch. Rational Mech. Anal. 124 (1993), 43-65.

[EZ] M. Escobedo and E. Zuazua, Large time behaviour for convection-diffusion equations in $\mathbb{R}^{n}$, J. Funct. Anal. 100 (1991), 119-161.

[GvDD] R. E. Grundy, C. J. van Duijn, and C. N. Dawson, Asymptotic profiles with finite mass in one-dimensional contaminant transport through porous media: The fast reaction case, Q. Jl Mech. Appl. Math. 47 (1994), 69-106.

[KaTz] M. A. Katsoulakis and A. E. Tzavaras, Contractive Relaxation Systems and the Scalar Multidimensional Conservation Law, Comm. P.D.E. 22 (1997), 195-233.

[Ko] V. Komornik, Exact Controllability and Stabilization. The Multiplier Method, RAM Res. Appl. Math. 36, Masson/Wiley, Paris/Chichester, 1994.

[KT] A. Kurganov and E. Tadmor, Stiff systems of hyperbolic conservation laws: Convergence and error estimates, SIAM J. Math. Anal. 28 (1997), 1446-1456.

[LSU] O. A. Ladyženskaja, V. A. Solonnikov, and N. N. Ural'ceva, Linear and Quasilinear Equations of Parabolic Type, Transl. Math. Monogr. 23, Amer. Math. Soc., Providence, 1968.

[LS] Ph. Laurençot and F. Simondon, Long-time behaviour for porous medium equations with convection, Proc. Roy. Soc. Edinburgh Sect. A 128 (1998), 315-336.

[LN] H. Liu and R. Natalini, Long-time diffusive behaviour of solutions to a hyperbolic relaxation system, Asymptot. Anal. 25 (2001), 21-38.

[L] T.-P. Liu, Hyperbolic conservation laws with relaxation, Comm. Math. Phys. 108 (1987), 153175.

[LP] T.-P. Liu and M. Pierre, Source-solutions and asymptotic behaviour in conservation laws, J. Differential Equations 51 (1984), 419-441.

[Na] R. Natalini, Recent results on hyperbolic relaxation problems, in "Analysis of Systems of Conservation Laws," Chapman \& Hall/CRC, Boca Raton, 1999, pp. 128-198.

[Re] G. Reyes, Asymptotic behaviour in convection-diffusion processes, Nonlinear Anal. 37 (1999), 301-318.

[Si] J. Simon, Compact sets in the space $L^{p}(0, T ; B)$, Ann. Mat. Pura Appl. (4) 146 (1987), 65-96.

[TW] A. Tveito and R. Winther, On the rate of convergence to equilibrium for a system of conservation laws including a relaxation term, SIAM J. Math. Anal. 28 (1997), 136-161.

[Va] J. L. Vazquez, Asymptotic behaviour of nonlinear parabolic equations. Anomalous exponents, in "Degenerate Diffusions," W. M. Ni, L. A. Peletier \& J. L. Vazquez (eds.), IMA Vol. Math. Appl. 47, Springer, New York, 1993, pp. 215-228.

[Zu] E. Zuazua, Weakly nonlinear large time behavior in scalar convection-diffusion equations, Differential Integral Equations 6 (1993), 1481-1491. 